\section{Microelectronics Packaging Handbook}

Edited by Rao R. Tummala and

Eugene J. Rymaszewski;

(Van Nostrand Reinhold, New York, 1989), approx. 1160 pages.

ISBN: 0-442-20578-3

The Microelectronics Packaging Handbook represents a massive and successful effort by an experienced group of IBM scientists and engineers with excellent credentials to compile and present the relevant design and development work in microelectronics packaging. Major topics are treated in individual chapters to a reasonable level of technical depth, and extensive reference lists are provided at the end of each chapter. However, the chapters are not isolated; intra-chapter cross referencing has been provided, attributable to thorough editing. This reference will be of equal value to the packaging technologist, the systems user of packaged electronics and, perhaps of greatest importance, the device designer.

The material covering electrical design and performance (chapters 2 and 3) provide useful insights into the extensive efforts that IBM has devoted to the layout and modeling of complex interconnect systems. The thermal management section reviews the basic concepts of heat transfer in simple terms. They briefly discuss the modeling tools as well as the experimental methods for package thermal characterization. Emphasis, again, is on complex, multi-chip applications and the impacts of material, geometrical and environmental variables on thermal characteristics are neglected.

The mechanical analysis section provides the basic strength of materials equations which exhibit the key material and design variables. Needed material properties are tabulated for easy reference and graphs comparing measurements and predictions enable the reader to understand the limitations of the models. Examples include solder fatigue in the joints of leadless ceramic chip carriers and in flip chips, where extensive use is made of the modified Coffin-Manson relations; and thermally induced die fracture.

A brief treatment of reliability issues includes an excellent review of reliability methodologies, specific acceleration factors and a useful discussion of the chemical and electrochemical principles applied to corrosion and related failure rate predictions.

The chapter on molding compounds provided a good, general overview, although there are a few omissions. For example, they fail to address the latest developments with low stress modifiers or other specialty materials (e.g., low for memory components). The descriptions for the materials which constitute a molding compound are good. Also, though brief, there is a good description of the properties important in processing and how processing parameters affect the quality of the part. Extensive treatment is given to wire sweep and issues related to molding stresses. Finally, there is a good overview on less traditional coatings and processes (glob top and reaction injection molding). Treatments of the role of fillers and filler/resin interactions, and resin rheology and curing characteristics are very good.

The sections on chip/package and package/board interconnection provided brief overviews of the diverse technologies which would be of great use to the uninitiated - again, the extensive reference list is of value. Use of excellent diagrams and descriptive material and detailed discussion of critical materials and design issues highlight these sections. The discussion of SMT is particularly helpful.

The final sections of the book provide an overview of the range of applications. These sections are useful for background, but give no detailed technical insights into the trade-offs implied in the examples.

There is room for improvement, even in a handbook of this caliber. References should be updated; there are almost no citations of papers published since 1986. Certain current topics should also be added: compliant leads and their effects on solder fatigue; and preconditioning of plastic packages are two examples. Finally, results of FEA models for the electrical, thermal and mechanical analysis of packages should be included as this tool is now commonly used in the industry.

Reviewer: Robert C. Sundahl is manager of technology development, New Packaging Technology Development Group, Intel Corporation, Chandler, AZ. Also contributing to the review were K. Kinsman, B. Jahsman, N. Grayeli, C. Steidel, S. Prough, and M. Aghazadeh.

\section{Nonlinear Phenomena in Materials Science}

Edited by L.P. Kubin and G. Martin

(Trans Tech Publications, Switzerland, 1988), approx. 500 pages.

ISBN: 0-87849-565-7

This soft-backed bound volume is the proceedings of an international CNPS meeting held in Aussois, September 1987. Its stated objective is to be "of some help to those interested in a synthesis of present achievements and potential developments in the understanding of nonlinear phenomena in materials science." This could be taken to include the assimilation of the spectacular recent developments in the understanding of nonlinear dynamical systems into the materials field.

The 45 contributed papers, some from very eminent authors, are divided into four sections. Almost all the contributions are theoretical.

The first section, entitled "Basics," provides an introduction to modern analysis techniques such as the use of phase portraits, the concept of attractors and transitions to chaos, weakly nonlinear systems and the Ginsberg-Landau equation, together with stochastic models. Not until well past page 100 do we first meet any materials science. It is a measure of the embryonic nature of this field, and perhaps therefore the need for such conferences as this one, that one searches the rest of the volume almost in vain to find these techniques being used!

The second section contains just three papers on solidification. These include an excellent contribution from B. Caroli, C. Caroli and B. Roulet on "The Thermodynamics of Solidification and Kinetic Effects" in which they develop a phenomological irreversible thermodynamic model describing uniform interface kinetics in binary mixtures. This is used to derive conditions under which solute trapping becomes possible and to critically analyse the models of Aziz and Jackson et al., which were developed to describe segregation behaviour under laser annealing conditions. Finally they propose a new model which would give rise to oscillatory modes of growth.

The third section on solid-state reactions and irradiation effects includes papers on phase separation and the effect of elastic stresses on both thermodynamic and kinetic properties. F.C. Larche, in a chapter on "Thermodynamics of Stressed Solids", shows that, under specific conditions, elastic energy can behave as an excess free energy and can be accommodated within the framework of classical thermodynamics. However, in general, due to the longrange nature of elastic forces, sample shape effects become important and local analysis is inappropriate. The bulk of this section, however, is devoted to irradiation effects, including mechanisms for dislocation pattern formation during irradiation.

This theme of dislocation patterning is continued in section four, where plastic instabilities are also considered. The chapter by G. Ananthakrishna on "Dislocation Dynamics and Cooperative Behaviour of Dislocations" is unique in the volume in its use of phase portraits to illustrate the transition to chaotic dynamics exhibited by his model. The volume ends with two more peripheral papers, one on charge density waves and a paper in which analogies are 
drawn between the onset of spatiotemporal chaos in a convecting fluid and the martensitic transformation.

The potential for applying the new techniques of nonlinear dynamical systems analysis to the materials science field is clearly great. As J. Friedel notes in his preface to the volume, one of the main characteristics of materials science is that it deals with properties which often deviate easily from the continuous linearised approximation, either because the speed of a linear reaction can be varied over a very large range or because one is near to an abrupt change of some dynamic state of self organisation. The contents of the volume show how little appreciated this potential is as yet.

Hopefully the first section of this book will stimulate materials scientists studying nonlinear systems to learn more about these new techniques. This volume will give them no more than a fairly superficial introduction, but if it generates such an interest then the next conference on this subject should achieve a closer meeting of minds than this reviewer imagines took place at Aussois.

Reviewer: D.T.J. Hurle is a special research officer at Royal Signals and Radar Establishment. His principal interest is in the crystal growth of electronic materials.

\section{Superalloys, Supercomposites and Superceramics \\ Edited by John K. Tien and Thomas Caulfield}

(Academic Press, 1989), 755 pages. ISBN: 0-12-690845-1

This book is a recent addition to the Materials Science Series published by Academic Press and consists of 22 chapters authored by experts in their specialties. It should be viewed primarily as a sampling of contemporary subjects in superalloy technology, insofar as 17 of the 22 chapters deal with various aspects of superalloy research and technology. It appears that no special attempt was made by the editors to cross reference the various chapters in order to make the book more useful. While reading the book, I was constantly comparing it to the two existing volumes The Superalloys (1972: C.T. Sims and W.C. Hagel, editors) and the recently updated volume Superalloys II (1987: C.T. Sims, N.S. Stoloff, and W.C. Hagel, editors). These two volumes attempt to give a broad overview of the various important aspects of superalloy technology; and while they have some inadequacies, each volume by itself presents a comprehensive view of superalloys technology aimed at a broad audience. Unfortunately, despite a few strong chapters, Superalloys, Supercomposites and Superceramics as a whole does not appear to be as useful as the above two existing superalloy volumes.

Specific chapters on superalloy technology are at the same level of quality as the Sims et al. volumes. The chapters on melt processing, casting of superalloy components, single crystal superalloys, and thermomechanical processing of superalloys, are all pretty solid papers. Similarly, the chapters on cyclic deformation of superalloys, high temperature oxidation, hydrogen embrittlement of superalloys, and intermetallic compounds, are also quite comprehensive and are worthy of the reader's attention. The final four chapters on superalloy composites, ceramics and carbon-carbon composites are concise treatments, but the authors have apparently done a good job of headlining the most important comparisons of these materials to superalloys.

The most glaring problem is that there is no chapter which deals directly with "alloy design" as applied to superalloys. I am referring to the analytical methodologieswhich have become increasingly sophisticated' during the last decade-of predicting phase stability in superalloys. The earliest versions of these phase compatibility calculations were known as PHACOMP, ${ }^{2}$ and were developed in the $1960 \mathrm{~s}$ in order to have a tool to predict which superalloy compositions would lead to the existence of brittle, topologically closed packed (TCP) phases such as sigma, laves, etc. which can lead to severe degradation in ductility. The book's virtual lack of any treatment of phase stability methods becomes apparent in the eleventh chapter "Creep and Stress Rupture - Long Term." The half page in chapter eleven on PHACOMP and similar methodologies is not enough to explain the importance of these techniques to superalloy technology or, in fact, explain to the reader the content of three figures in the chapter. An entire chapter on phase stability would certainly have been more useful than the chapter on modeling of ternary phase compatibility.

Unfortunately, other important superalloy subjects are not handled comprehensively. A case in point is the chapter on "Oxide Dispersion Strengthened (ODS) Alloys" (Chapter 9). This is doubly disconcerting insofar as the chapter will also be appearing in a forthcoming book The Handbook of Metallic Composites. The authors begin by implying that the only useful ODS alloys are the mechanically alloyed "MA" series marketed by INCO. However, a large amount of understanding and some engine experience was obtained prior to the advent of mechanical alloying with the thoria-dispersed alloys TD-Ni and
TD-NiCr: these two alloys are indeed commercially available today ${ }^{3}$ and referred to later in the chapter. The authors' treatment of grain morphology/fracture and texture development in ODS alloys are both extremely limited. One suspects they intentionally chose to ignore the results of research groups at other institutions. For example, no mention is made of MA 754's tendency for intergranular fracture under long-term creep conditions at elevated temperatures, or the recent constrained cavity growth models which examine this problem. "Similarly, the lack of success in attaining a $<100>$ coarse-grained texture in the strongest ODS superalloy, MA $6000,5,6$ has been an impediment to this alloy's application. However, no mention is made of this problem. Although it is about five years old, the chapter on ODS alloys in Reference 6 provides a better overview of ODS alloys.

In summary, I would advise readers who are seeking a comprehensive treatment of superalloys to consult the two volumes by Sims et. al. For those who want to purchase a volume which examines recent trends in superalloys and future replacement materials, the most recent volume of the Seven Springs Superalloys Symposium would be a wise alternative.

\section{References}

1. M. Morinaga et al., "New Phacomp and its Applications to Alloy Design," Supemiloys 1984, edited by M. Gell et al. (TMS-AIME, 1984) p. 523-532.

2. C.T. Sims, "Prediction of Phase Composition" in Superalloys $1 l$, edited by C.T. Sims, N.S. Stoloff, and W.C. Hagel (John Wiley and Sons, 1987) p. $217-240$.

3. TD-Ni and TD-NiCr are still available from Sheritt-Gordon Mines, Ltd. of Canada in both powder and sheet form.

4. J.J. Stephens and W.D. Nix, "Constrained Cavity Growth Models of Longitudinal Creep Deformation of Oxide Dispersion Strengthened Alloys, Metallurgical Tmnsactions 17A (1986), p. 281-291.

5. T.E. Howson, D.A. Mervyn, and J.K. Tien "Creep and Stress Rupture of a Mechanically Alloyed Oxide Dispersion Strengthened and Precipitation Strengthened Ni Base Superalloy," Metallurgical Transactions 11A (1980), p. 16091616.

6. G.H. Gessinger, Pouder Metallurgy of Supemlloys (Butterworths, Ltd., 1984) p. 218.

7. D.N. Duhl, G. Maurer, S. Antolovich, C. Lund, and S. Reichman, Superalloys 1988 (TMS, 1988).

Reviewer: John Stephens is with the Physical Metallurgy Division of Sandia National Laboratories, Albuquerque, New Mexico. His research interests include structure/property relations in alloys at elevated temperatures. 